transplants were from living-related donors. At the time of transplantation the mean height $z$ score was $-1.15+/-1.7$ and BMI $z$ score was $0.44+/-1.8$. Eighteen months after transplantation, catch-up growth was seen in $40 \%$ of children, $30 \%$ had normal linear growth without any catch-up and $30 \%$ had slow growth rate after transplantation. Children with evidence of catch-up growth (growth velocity $z$ score $>0$ ) had more growth retardation at the time of transplantation, and were receiving lower doses of prednisone at 1.5 years after transplantation. Younger infants (below 6 months) were most likely to demonstrate catch-up growth after transplantation. In summary, a large proportion of children have growth retardation at the time of liver transplantation. Serum albumin increased significantly after $(39.8+/-5.2 \mathrm{~g} / \mathrm{L})$ vs before (34 +/-11g/L)transplantation, and Alanine transferase (ALT) decreased significantly from $(130+/-260 \mathrm{U} / \mathrm{L})$ to $(30+/-15 \mathrm{U} / \mathrm{L})$. Poor growth after transplantation occurred more in those receiving higher doses of corticosteroid. This growth retardation is inversely correlated with age. Growth after transplantation is proportional to the degree of growth retardation at transplantation and inversely correlated to age at transplantation.

\section{THE METABOLOMIC PROFILE OF UMBILICAL CORD BLOOD IN NEONATAL HYPOXIC ISCHAEMIC ENCEPHALOPATHY}

doi:10.1136/archdischild-2012-302724.0165

${ }^{1} \mathrm{BH}$ Walsh, ${ }^{2} \mathrm{D}$ Broadhurst, ${ }^{3} \mathrm{R}$ Mandal, ${ }^{3} \mathrm{DS}$ Wishart, ' $\mathrm{GB}$ Boylan, ${ }^{4} \mathrm{LC}$ Kenny, 'DM Murray. ${ }^{1}$ Neonatal Brain Research Group, Department of Paediatrics and Child Health, University College Cork, Cork, Ireland; '2Department of Medicine; ${ }^{3}$ Departments of Biological and Computing Sciences, University of Alberta, Edmonton, AB, Canada; ${ }^{4}$ Department of Obstetrics and Gynaecology, University College Cork, Cork, Ireland

Background Hypoxic ischaemic encephalopathy (HIE) is associated with the activation of multiple biochemical pathways. The importance of these pathways individually, and that of their interaction, in the disease process is not fully understood. The aim of this study was to describe and quantify the metabolomic profile of umbilical cord blood samples in a carefully defined population of full-term infants with HIE.

Methods Full-term infants with perinatal asphyxia (with and without HIE) and healthy controls had umbilical cord blood drawn and bio-banked at $-80^{\circ} \mathrm{C}$, within 3 hours of birth. A combined direct injection and LC-MS/MS assay (AbsolutIDO p180 kit, Biocrates Life Sciences AG, Innsbruck, Austria) was used for the metabolomic analyses of the samples. The degree of encephalopathy among those with asphyxia was defined using both continuous multichannel-EEG in the first 24 hours, and modified Sarnat score.

Results 142 neonates were included in the analysis $(\mathrm{HIE}=31$, asphyxia without encephalopathy $=40$, controls $=71$ ). There was a significant alteration $(\mathrm{p}<0.01)$ in 29 metabolites from 3 distinct metabolite classes (Amino Acids, Carnitines, and Phosphatidylcholines) between study groups. 13 of these metabolites were significantly altered between HIE and controls. Cross-validated Partial Least Square Discriminant Analysis models were developed to distinguish between the groups. The HIE model differentiated significantly between HIE, and those without HIE (AUC=0.93, $\mathrm{R}^{2}=0.36, \mathrm{O}^{2}=0.25$ ).

Conclusion The description of the metabolomic profile from umbilical cord plasma and the specific metabolite signature associated with HIE, offers insight into the disease mechanism and the possibility of an early screening test.

\section{SIMULTANEOUS ${ }^{31}$ P-MAGNETIC RESONANCE AND NEAR- INFRARED SPECTROSCOPIC INVESTIGATION OF BRAIN TISSUE OXYGENATION, CYTOCHROME-C-OXIDASE AND INTRACELLULAR METABOLITES DURING PERINATAL CEREBRAL HYPOXIA-ISCHAEMIA}

doi:10.1136/archdischild-2012-302724.0166
II Tachtsidis, ${ }^{2} \mathrm{~A}$ Bainbridge, ${ }^{3} \mathrm{~S}$ Faulkner, ${ }^{2} \mathrm{D}$ Price, ${ }^{3} \mathrm{E}$ Powell, ${ }^{4} \mathrm{D}$ Thomas, ${ }^{2} \mathrm{E}$ Cady, ${ }^{3} \mathrm{~N}$ Robertson, ${ }^{\mathrm{X} X}$ Golay. ${ }^{\prime}$ Medical Physics and Bioengineering, University College London; ${ }^{2}$ Medical Physics and Bioengineering, University College London Hospital; '3nstitute for Women's Health; "Institute of Neurology, University College London, London, UK

Background Hypoxic-ischaemic neonatal encephalopathy is associated with high mortality and morbidity rates worldwide.

Aims To investigate brain haemodynamic, cytochrome-c-oxidase (CCO) and energy-resource changes during transient hypoxia-ischaemia (HI) and recovery using simultaneous broadband near-infrared spectroscopy (NIRS) and phosphorus $\left({ }^{31} \mathrm{P}\right)$ magnetic resonance spectroscopy (MRS).

Methods Nine healthy piglets (aged $<24 \mathrm{hr}$ ) were anaesthetised and physiologically monitored. Transient cerebral HI (duration 20 minutes) was induced by reducing the inspired oxygenation and reversibly inflating bilateral carotid artery occluders. Using ${ }^{31} \mathrm{P}$ MRS we measured inorganic phosphate ( $\mathrm{Pi}) / e p p$, phosphocreatine ( $\mathrm{PCr}$ )/epp, and nucleotide triphosphate (NTP)/epp where epp=exchangeable phosphate pool=Pi+PCr+3NTP. NIRS measured cerebral concentration changes of oxy-haemoglobin $\left(\mathrm{HbO}_{2}\right)$ and deoxy-haemoglobin $(\mathrm{HHb})$, and cytochrome-c-oxidase oxidation state changes ( $\Delta[\mathrm{oxCCO}])$.

Results Simultaneous ${ }^{31} \mathrm{P}-\mathrm{MRS}$ and NIRS results are shown. HI rapidly reduced brain oxygenation as shown by changes in haemoglobin difference $\left.\left(\Delta[\mathrm{Hbdiff}]=\Delta\left[\mathrm{HbO}_{2}\right]-\Delta[\mathrm{HHb}]\right)\right)$ closely followed by a fall in $\Delta$ [oxCCO]. PCr/epp fell, and $\mathrm{Pi} /$ epp rose, quickly while NTP/epp was buffered initially and only declined when $\Delta[\mathrm{oxCCO}]$ was significantly lowered.

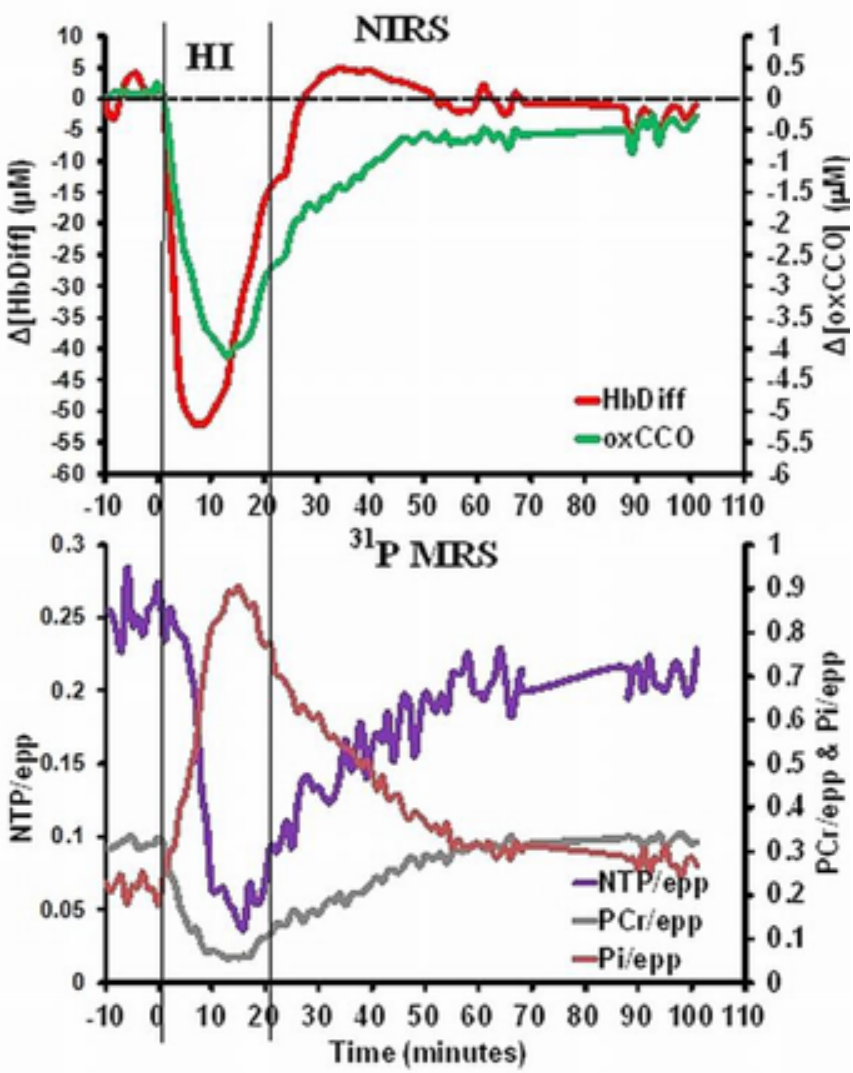

Abstract 166 Figure 1

Discussion During transient $\mathrm{HI}, \mathrm{CCO}$ becomes reduced due to oxygen depletion; adenosine triphosphate levels are initially preserved by the creatine kinase reaction leading to PCr decline whereas energy utilisation without oxidative phosphorylation leads to increased Pi. Complementary MRS and NIRS enable better understanding of the cerebral metabolic response to $\mathrm{HI}$ and can help evaluate early interventional therapies. 